\title{
EFFECTIVE ESTIMATES FOR BOUNDARY VALUE PROBLEMS
}

\author{
GOU-SHENG YANG AND JYH-WEN LEU
}

Abstract. We establish some effective estimates for the boundary value problems $x^{(6)}(t)-\sum_{i=0}^{5} p_{i}(t) x^{(i)}(t)=0, \quad x(a)=x^{\prime}(a)=x^{\prime \prime}(a)=x^{\prime \prime \prime}(a)=x^{(4)}(a)=x(b)=0$ and $x^{(6)}(t)-\sum_{i=0}^{5} p_{i}(t) x^{(i)}(t)=0, \quad x(a)=x^{\prime}(a)=x^{\prime \prime}(a)=x(b)=x^{\prime}(b)=x^{\prime \prime}(b)=0$.

\section{Introduction}

Bogar and Gustafson ([1]) have shown that the homogeneous bundary value problem

$$
\begin{gathered}
x^{(6)}-\sum_{i=0}^{2} p_{i}(t) x^{(i)}=0 ; \\
x(a)=x^{\prime}(a)=x^{\prime \prime}(a)=x^{\prime \prime \prime}(a)=x(b)=x^{\prime}(b)=0 ;
\end{gathered}
$$

where $p_{i} \in C[a, b], 0 \leq i \leq 2$, has only the trivial solution provided the inequality

$$
\frac{377.4}{10^{6}}(b-a)^{6}\left\|p_{0}\right\|+\frac{156.91}{10^{5}}(b-a)^{5}\left\|p_{1}\right\|+\frac{9}{2048}(b-a)^{4}\left\|p_{2}\right\|<1 ;
$$

is satisfied, where $\left\|p_{i}\right\|=\sup \left(\left|p_{i}(t)\right|, t \in[a, b]\right)$. For the complete differential equation

$$
x^{(6)}-\sum_{i=0}^{5} p_{i}(t) x^{(i)}=0,
$$

Received June 30, 1993, revised October 27, 1993.

1991 Mathematics Subject Classification. 34B15.

Key words and phrases. Boundary value problem, trivial solution. 
where $p_{i} \in C[a, b], 0 \leq i \leq 5$, Agarwal and Milovanovic ([2]) have shown that the boundary value problem (4),(2) has only the trivial solution provided the inequality

$$
\begin{gathered}
\frac{1}{32805}(b-a)^{6}\left\|p_{0}\right\|+\frac{25+34 \sqrt{10}}{911250}(b-a)^{5}\left\|p_{1}\right\|+\frac{1}{360}(b-a)^{4}\left\|p_{2}\right\| \\
+\frac{1}{30}(b-a)^{3}\left\|p_{3}\right\|+\frac{1}{5}(b-a)^{2}\left\|P_{4}\right\|+\frac{2}{3}(b-a)\left\|p_{5}\right\|=\theta<1
\end{gathered}
$$

is satisfied.

The main purpose of this paper is to obtain a similar result of Milovanovic in considering the boundary conditions

$$
\begin{gathered}
x(a)=x^{\prime}(a)=x^{\prime \prime}(a)=x^{\prime \prime \prime}(a)=x^{(4)}(a)=x(b)=0 ; \\
x(a)=x^{\prime}(a)=x^{\prime \prime}(a)=x(b)=x^{\prime}(b)=x^{\prime \prime}(b)=0 ;
\end{gathered}
$$

respectively, instead of the boundary conditions(2).

\section{Main result}

Theorem. A. The boundary value problem (4),(6) has only the trivial solution provided the inequality

$$
\begin{aligned}
& \frac{625}{6718464}(b-a)^{6}\left\|p_{0}\right\|+\frac{1}{720}(b-a)^{5}\left\|p_{1} \mid+\frac{1}{72}(b-a)^{4}\right\| p_{2} \| \\
& \quad+\frac{1}{12}(b-a)^{3}\left\|p_{3}\right\|+\frac{1}{3}(b-a)^{2}\left\|p_{4}\right\|+\frac{5}{6}(b-a)\left\|p_{5}\right\|=\theta_{1}<1
\end{aligned}
$$

is satisfied.

Theorem B. The boundary value problem (4),(7) has only the trivial solution provided the inequality

$$
\begin{aligned}
& \frac{1}{46080}(b-a)^{6}\left\|p_{p}\right\|+\frac{159+76 \sqrt{6}}{3000000}(b-a)^{5}\left\|p_{1}\right\|+\frac{1}{480}(b-a)^{4}\left\|p_{2}\right\| \\
& +\frac{41+28 \sqrt{7}}{9720}(b-a)^{3}\left\|p_{3}\right\|+\frac{13}{120}(b-a)^{2}\left\|p_{4}\right\|+\frac{1}{2}(b-a)\left\|p_{5}\right\|<1
\end{aligned}
$$

is satisfied.

For the proof of the theorems, we need the following:

Lemma 1 .Any function $x \in C^{(6)}[0,1]$ satisfying the conditions

$$
x(0)=x^{\prime}(0)=x^{\prime \prime}(0)=x^{\prime \prime \prime}(0)=x^{(4)}(0)=x(1)=0 ;
$$


can be written as

$$
x(t)=t^{4}(1-t) F(t)
$$

where

$$
\begin{array}{ll}
F(t)=\int_{0}^{t} \frac{F_{1}\left(t_{1}\right)}{t_{1}^{2}} d t_{1}, & F_{1}(t)=\int_{0}^{t} \frac{F_{2}\left(t_{2}\right)}{t_{2}^{2}} d t_{2}, \\
F_{2}(t)=\int_{0}^{t} \frac{F_{3}\left(t_{3}\right)}{t_{3}^{2}} d t_{3}, & F_{3}(t)=\int_{0}^{t} \frac{F_{4}\left(t_{4}\right)}{t_{4}^{2}} d t_{4}, \\
F_{4}(t)=\int_{1}^{t} \frac{t_{5}^{4}}{\left(1-t_{5}\right)^{6}} F_{5}\left(t_{5}\right) d t_{5}, & F_{5}(t)=\int_{1}^{t}\left(1-t_{6}\right)^{5} x^{(6)}\left(t_{6}\right) d t_{6} .
\end{array}
$$

proof.

Let $\phi(t)$ be the right hand side of (11) and satisfying the condition (10). Then

$$
\begin{aligned}
\phi(t)= & t^{4}(1-t) F(t) \\
\phi^{\prime}(t)= & {\left[4 t^{3}(1-t)-t^{4}\right] F(t)+t^{2}(1-t) F_{1}(t) } \\
\phi^{\prime \prime}(t)= & {\left[12 t^{2}(1-t)-8 t^{3}\right] F(t)+\left[6 t(1-t)-2 t^{2}\right] F_{1}(t)+(1-t) F_{2}(t) } \\
\phi^{\prime \prime \prime}(t)= & {\left[24 t(1-t)-36 t^{3}\right] F(t)+[18(1-t)-18 t] F_{1}(t) } \\
& +\left[\frac{6(1-t)}{t}-3\right] F_{2}(t)+\left[\frac{1-t}{t^{2}}\right] F_{3}(t) \\
\phi^{(4)}(t)= & {[24(1-t)-96 t] F(t)+\left[\frac{24(1-t)}{t}-72\right] F_{1}(t) } \\
& +\left[\frac{12(1-t)}{t^{2}}-\frac{24}{t}\right] F_{2}(t)+\left[\frac{4(1-t)}{t^{3}}-\frac{4}{t_{2}}\right] F_{3}(t) \\
& +\left[\frac{1-t}{t_{4}}\right] F_{4}(t) \\
\phi^{(5)}(t)= & {[-120] F^{\prime}(t)+\left[-120 t^{-1}\right] F_{1}(t)+\left[-60 t^{-2}\right] F_{2}(t) } \\
& +\left[-20 t^{-3}\right] F_{3}(t)+\left[-5 t^{-4}\right] F_{4}(t)+\left[(1-t)^{-5}\right] F_{5}(t) \\
\phi^{(6)}(t)= & {\left[\frac{-120}{t^{2}}\right] F_{1}(t)+\left[\frac{120}{t^{2}}\right] F_{1}(t)+\left[\frac{-120}{t^{3}}\right] F_{2}(t)+\left[\frac{120}{t^{3}}\right] F_{2}(t) } \\
& +\left[\frac{-60}{t^{4}}\right] F_{3}(t)+\left[\frac{60}{t^{4}}\right] F_{3}(t)+\left[\frac{-20}{t^{5}}\right] F_{4}(t)+\left[\frac{20}{t^{5}}\right] F_{4}(t) \\
& +\left[\frac{-5}{(1-6)^{6}}\right] F_{5}(t)+\left[\frac{5}{(1-t)^{6}}\right] F_{5}(t)+\left[x^{(6)}(t)\right]=x^{(6)}(t)
\end{aligned}
$$

It follows that

$$
\phi(t)=x(t)+\frac{1}{120} c_{1} t^{5}+\frac{1}{24} c_{2} t^{4}+\frac{1}{6} c_{3} t^{3}+\frac{1}{2} c_{4} t^{2}+c_{5} t+c_{6}
$$

for some constants $c_{i}, 1 \leq i \leq 6$.

Since $\phi(t)$ satisfies (10), it follows that

$$
c_{1}=c_{2}=c_{3}=c_{4}=c_{5}=c_{6}=0,
$$

hence $\phi(t)=x(t)$. 
Lemma 2. Let $x$ be as in Lemma 1. Then

$$
\begin{aligned}
|x(t)| & \leq \frac{625}{6718464} M \\
\left|x^{\prime}(t)\right| & \leq \frac{1}{720} M \\
\left|x^{\prime \prime}(t)\right| & \leq \frac{1}{72} M \\
\left|x^{\prime \prime \prime}(t)\right| & \leq \frac{1}{12} M \\
\left|x^{(4)}(t)\right| & \leq \frac{1}{3} M \\
\left|x^{(5)}(t)\right| & \leq \frac{5}{6} M
\end{aligned}
$$

where $M=\max _{0 \leq t \leq 1}\left|x^{(6)}(t)\right|$

Proof. From(12), it is immediately that

$$
\begin{array}{ll}
\left|F_{5}(t)\right| \leq \frac{1}{6} M(1-t)^{6}, & \left|F_{4}(t)\right| \leq \frac{1}{30} M t^{5}, \\
\left|F_{3}(t)\right| \leq \quad \frac{1}{120} M t^{4}, & \left|F_{2}(t)\right| \leq \frac{1}{360} M t^{3}, \\
\left|F_{1}(t)\right| \leq \quad \frac{1}{720} M t^{2}, & |F(t)| \leq \frac{1}{720} M t .
\end{array}
$$

Thus, it follows from (11) and (18) that

$$
|x(t)| \leq \frac{1}{720} t^{5}(1-t) M .
$$

The right hand side of (19) attains its maximum at $t=\frac{2}{3}$, so that

$$
|x(t)| \leq \frac{625}{6718464} M, \quad 0 \leq t \leq 1 .
$$

It follows from (13) and (18) that

$$
\begin{aligned}
\left|x^{\prime}(t)\right| & \leq\left|4 t^{3}(1-t)-t^{4}\right| \frac{t}{720} M+\left|t^{2}(1-t)\right| \frac{t^{2}}{720} M \\
& =\frac{M}{720} t^{4}[(1-t)+|4-5 t|] \\
& =\frac{M}{720} \begin{cases}t^{4}(5-6 t), & 0 \leq t \leq \frac{4}{5}, \\
t^{4}(4 t-3), & \frac{4}{5} \leq t \leq 1 .\end{cases}
\end{aligned}
$$

The right hand side of (20) attains its maximum at $t=1$, so that

$$
\left|x^{\prime}(t)\right| \leq \frac{M}{720}, \quad 0 \leq t \leq 1 .
$$


From (14) and (18), we have

$$
\begin{aligned}
\left|x^{\prime \prime}(t)\right| & \leq\left|12 t^{2}(1-t)-8 t^{3}\right| \frac{t}{720} M+\left|6 t(1-t)-2 t^{2}\right| \frac{t^{2}}{720} M+|(1-t)| \frac{t^{3}}{360} M \\
& =\frac{M}{360} t^{3}[|6-10 t|+|3-4 t|+(1-t)] \\
& =\frac{M}{360} \begin{cases}t^{3}(10-15 t), & 0 \leq t \leq \frac{3}{5} \\
t^{3}(-2+5 t), & \frac{3}{5} \leq t \leq \frac{3}{4} \\
t^{3}(-8+13 t), & \frac{3}{4} \leq t \leq 1 .\end{cases}
\end{aligned}
$$

The right hand side of (21) attains its maximum at $t=1$, so that

$$
\left|x^{\prime \prime}(t)\right| \leq \frac{M}{72}, \quad 0 \leq t \leq 1
$$

From (15) and (18), we have

$$
\begin{aligned}
\left|x^{\prime \prime \prime}(t)\right| \leq & \left|24 t(1-t)-36 t^{2}\right| \frac{t}{720} M+|18(1-t)-18 t| \frac{t^{2}}{720} M \\
& +\left|\frac{6(1-t)}{t}-3\right| \frac{t^{3}}{360} M+\left|\frac{1-t}{t^{2}}\right| \frac{t^{4}}{120} M \\
= & \frac{M}{120} t^{2}[2|2-5 t|+3|1-2 t|+|2-3 t|+(1-t)] \\
= & \frac{M}{120} \begin{cases}t^{2}(10-20 t), & 0 \leq t \leq \frac{2}{5} \\
2 t^{2}, & \frac{2}{5} \leq t \leq \frac{1}{2} \\
t^{2}(-8+12 t), & \frac{1}{2} \leq t \leq \frac{2}{3} \\
t^{2}(-8+18 t), & \frac{2}{3} \leq t \leq 1\end{cases}
\end{aligned}
$$

The right hand side of (22) attains its maximum at $t=1$, so that

$$
\left|x^{\prime \prime \prime}(t)\right| \leq \frac{M}{12}, \quad 0 \leq t \leq 1
$$

From (16) and (18), we have

$$
\begin{aligned}
\left|x^{(4)}(t)\right| \leq & |24 t(1-t)-96 t| \frac{t}{720} M+\left|\frac{24(1-t)}{t}-72\right| \frac{t^{2}}{720} M+\left|\frac{12(1-t)}{t^{2}}-\frac{24}{t}\right| \frac{t^{3}}{360} M \\
& +\left|\frac{4(1-t)}{t^{3}}-\frac{4}{t^{2}}\right| \frac{t^{4}}{120} M+\left|\frac{1-t}{t^{4}}\right| \frac{t^{5}}{30} M \\
= & \frac{M}{30} t[|1-5 t|+|1-4 t|+|1-3 t|+|1-2 t|+(1-t)] \\
= & \frac{M}{30} \begin{cases}t(5-15 t), \quad 0 \leq t \leq \frac{1}{5} \\
t(3-5 t), \quad \frac{1}{5} \leq t \leq \frac{1}{4} \\
t(1+3 t), & \frac{1}{4} \leq t \leq \frac{1}{3} \\
t(-1+9 t), & \frac{1}{3} \leq t \leq \frac{1}{2} \\
t(-3+13 t), & \frac{1}{2} \leq t \leq 1\end{cases}
\end{aligned}
$$


The right hand side of (23) attains its maximum at $t=1$, so that

$$
\left|x^{(4)}(t)\right| \leq \frac{M}{3}, \quad 0 \leq t \leq 1 .
$$

Finally, from (17) and (18), we have

$$
\begin{aligned}
\left|x^{(5)}(t)\right| \leq & 120 \frac{t}{720} M+\frac{120}{t} \frac{t^{2}}{720} M+F \frac{60}{t^{2}} \frac{t^{3}}{360} M+\frac{20}{t^{3}} \frac{t^{4}}{120} M \\
& +\frac{5}{t^{4}} \frac{t^{5}}{30} M+\frac{1}{(1-t)^{5}} \frac{1}{6}(1-t)^{6} M \\
= & \frac{M}{6}(1+4 t), \quad 0 \leq t \leq 1 .
\end{aligned}
$$

The right hand side of (24) attains its maximum at $t=1$, so that

$$
\left|x^{(5)}(t)\right| \leq \frac{5}{6 M}, \quad 0 \leq t \leq 1 .
$$

This completes the proof of Lemma 2.

Lemma 3. Let $x \in C^{(6)}[a, b]$, and satisfy the conditions (6). Then

$$
\begin{aligned}
|x(t)| & \leq \frac{625}{6718464}(b-a)^{6} \mu, \\
\left|x^{\prime}(t)\right| & \leq \frac{1}{720}(b-a)^{5} \mu, \\
\left|x^{\prime \prime}(t)\right| & \leq \frac{1}{72}(b-a)^{4} \mu, \\
\left|x^{\prime \prime \prime}(t)\right| & \leq \frac{1}{12}(b-a)^{3} \mu, \\
\left|x^{(4)}(t)\right| & \leq \frac{1}{3}(b-a)^{2} \mu, \\
\left|x^{(5)}(t)\right| & \leq \frac{5}{6}(b-a) \mu,
\end{aligned}
$$

where $\mu=\max _{a \leq t \leq b}\left|x^{(6)}(t)\right|$. The inequalities are the best possible as the identity holds for the function $x(t)=(t-a)^{5}(b-t)$.

Proof. The proof requires only the transformation $\mu=a+(b-a) t, 0 \leq t \leq 1$, in Lemma, 2.

Lemma 4. Any function $x \in C^{(6)}[0,1]$ satisfying the conditions

$$
x(0)=x^{\prime}(0)=x^{\prime \prime}(0)=x(1)=x^{\prime}(1)=x^{\prime \prime}(1)=0
$$

can be written as

$$
x(t)=t^{2}(1-t)^{3} G(t),
$$


where

$$
\begin{array}{ll}
G(t)=\int_{0}^{t} t_{1}^{-2} G_{1}\left(t_{1}\right) d t_{1}, & G_{1}(t)=\int_{0}^{t} t_{2}^{-2} G_{2}\left(t_{2}\right) d t_{2}, \\
G_{2}(t)=\int_{1}^{t} t_{3}^{2}\left(1-t_{3}\right)^{-4} G_{3}\left(t_{3}\right) d t_{3}, & G_{3}(t)=\int_{1}^{t}\left(1-t_{4}\right)^{-2} G_{4}\left(t_{4}\right) d t_{4}, \\
G_{4}(t)=\int_{1}^{t}\left(1-t_{5}\right)^{-2} G_{5}\left(t_{5}\right) d t_{5}, & G_{5}(t)=\int_{1}^{t}\left(1-t_{6}\right)^{5} x^{(6)}\left(t_{6}\right) d t_{6}
\end{array}
$$

proof. Let $\psi(t)$ be the right hand side of (26) and satisfying the condition (25). Then

$$
\begin{aligned}
& \psi(t)=t^{2}(1-t)^{3} G(t) \\
& \psi^{\prime}(t)=\left[2 t(1-t)^{3}-3 t^{2}(1-t)^{2}\right] G(t)+\left[(1-t)^{3}\right] G_{1}(t) \\
& \psi^{\prime \prime}(t)=\left[2(1-t)^{3}-12 t(1-t)^{2}+6 t^{2}(1-t)\right] G(t)+\left[\frac{2(1-t)^{3}}{t}-6(1-t)^{2}\right] G_{1}(t) \\
& +\left[\frac{(1-t)^{3}}{t}\right] G_{2}(t) \\
& \psi^{\prime \prime \prime}(t)=\left[-18(1-t)^{2}+36 t(1-t)-6 t^{2}\right] G(t)+\left[\frac{-18(1-t)^{2}}{t}+18(1-t)\right] G_{1}(t) \\
& +\left[\frac{-9(1-t)^{2}}{t^{2}}\right] G_{2}(t)+\left[\frac{1}{1-t}\right] G_{3}(t) \\
& \left.\psi^{(4)}(t)=[72(1-t)-48 t] G(t)\right]+\left[\frac{72(1-t)}{t}-24\right] G_{1}(t)+\left[\frac{36(1-t)}{t^{2}}\right] G_{2}(t) \\
& +\left[\frac{-8}{(1-t)^{2}}\right] G_{3}(t)+\left[\frac{1}{(1-t)^{3}}\right] G_{4}(t) \\
& \psi^{(5)}(t)=[-120] G(t)+\left[\frac{-120}{t}\right] G_{1}(t)+\left[\frac{-60}{t^{2}}\right] G_{2}(t)+\left[\frac{20}{(1-t)^{3}}\right] G_{3}(t) \\
& +\left[\frac{-5}{(1-t)^{4}}\right] G_{4}(t)+\left[\frac{1}{(1-t)^{5}}\right] G_{5}(t) \\
& \psi^{(6)}(t)=\left[\frac{-120}{t^{2}}\right] G_{1}(t)+\left[\frac{120}{t^{2}}\right] G_{1}(t)+\left[\frac{-120}{t^{3}}\right] G_{2}(t)+\left[\frac{120}{t^{3}}\right] G_{2}(t) \\
& +\left[\frac{-60}{(1-t)^{4}}\right] G_{3}(t)+\left[\frac{60}{(1-t)^{4}}\right] G_{3}(t)+\left[\frac{-20}{(1-t)^{5}}\right] G_{4}(t)+\left[\frac{20}{(1-t)^{5}}\right] G_{4}(t) \\
& +\left[\frac{-5}{(1-t)^{6}}\right] G_{5}(t)+\left[\frac{5}{(1-t)^{6}}\right] G_{5}(t)+x^{(6)}(t) \\
& =x^{(6)}(t) \text {. }
\end{aligned}
$$

It follows that

$$
\psi(t)=x(t)+\frac{1}{120} c_{1} t^{5}+\frac{1}{24} c_{2} t^{4}+\frac{1}{6} c_{3} t^{3}+\frac{1}{2} c_{4} t^{2}+c_{5} t+c_{6}
$$

for some constants $c_{i}, 1 \leq i \leq 6$. 
Since $\psi(t)$ satisfies (25), we find that

$$
c_{1}=c_{2}=c_{3}=c_{4}=c_{5}=c_{6}=0 .
$$

Hence $\psi(t)=x(t)$.

Lemma 5. Let $x$ be as in Lemma 4. Then

$$
\begin{aligned}
|x(t)| & \leq \frac{1}{46080} M, \\
\left|x^{\prime}(t)\right| & \leq \frac{159+76 \sqrt{6}}{3000000} M, \\
\left|x^{\prime \prime}(t)\right| & \leq \frac{1}{480} M, \\
\left|x^{\prime \prime \prime}(t)\right| & \leq \frac{41+28 \sqrt{7}}{9720} M, \\
\left|x^{(4)}(t)\right| & \leq \frac{13}{120} M, \\
\left|x^{(5)}(t)\right| & \leq \frac{1}{2} M,
\end{aligned}
$$

where $M=\max _{0 \leq t \leq 1}\left|x^{(6)}(t)\right|$.

proof. From (27), it is immediately that

$$
\begin{array}{rlrl}
\left|G_{5}(t)\right| & \leq \frac{1}{6}(1-t)^{6} M, & & \left|G_{4}(t)\right| \leq \frac{1}{30}(1-t)^{5} M, \\
\left|G_{3}(t)\right| \leq \frac{1}{120}(1-t)^{4} M, & & \left|G_{2}(t)\right| \leq \frac{1}{360} t^{3} M, \\
\left|G_{1}(t)\right| \leq \frac{1}{720} t^{2} M, & & |G(t)| \leq \frac{1}{720} t M .
\end{array}
$$

Thus, it follows from (26) and (33) that

$$
|x(t)| \leq \frac{1}{720} t^{3}(1-t)^{3} M
$$

The right hand side of (34) attains its maximum at $t=\frac{1}{2}$, so that

$$
|x(t)| \leq \frac{1}{46080} M
$$

It follows from (28) and (33) that

$$
\begin{aligned}
\left|x^{\prime}(t)\right| & \leq\left|2 t(1-t)^{3}-3 t^{2}(1-t)\right| \frac{t}{720} M+\left|(1-t)^{3}\right| \frac{t^{2}}{720} M \\
& =\frac{M}{720} t^{2}(1-t)^{2}[|2-5 t|+(1-t)] \\
& =\frac{M}{720} \begin{cases}t^{2}(1-t)^{2}(3-6 t), & 0 \leq t \leq \frac{2}{5}, \\
t^{2}(1-t)^{2}(-1+4 t), & \frac{2}{5} \leq t \leq 1 .\end{cases}
\end{aligned}
$$


The right hand side of (35) attains its maximum at $t=\frac{4+\sqrt{6}}{10}$, so that

$$
\left|x^{\prime}(t)\right| \leq \frac{159+76 \sqrt{6}}{3000000} M, \quad 0 \leq t \leq 1 .
$$

From (29) and (33), we have

$$
\begin{aligned}
\left|x^{\prime \prime}(t)\right| \leq & \left|2(1-t)^{3}-12 t(1-t)^{2}+6 t^{2}(1-t)\right| \frac{t}{720} M \\
& +\left|\frac{2(1-t)^{3}}{t}-6(1-t)\right| \frac{t^{2}}{720} M+\left|\frac{(1-t)^{3}}{t^{2}}\right| \frac{t^{3}}{360} M \\
= & \frac{M}{360} t(1-t)\left[\left|10 t^{2}-8 t+1\right|+\left|4 t^{2}-5 t+1\right|+\left(t^{2}-2 t+1\right)\right] \\
= & \frac{M}{360}\left\{\begin{array}{l}
t(1-t)\left(15 t^{2}-15 t+3\right), \quad 0 \leq t \leq \frac{4-\sqrt{6}}{10} \\
t(1-t)\left(-5 t^{2}+t+1\right), \quad \frac{4-\sqrt{6}}{10} \leq t \leq \frac{1}{4}, \\
t(1-t)\left(-13 t^{2}+11 t-1\right), \frac{1}{4} \leq t \leq \frac{4+\sqrt{6}}{10} \\
t(1-t)\left(7 t^{2}-5 t+1\right), \quad \frac{4+\sqrt{6}}{10} \leq t \leq 1,
\end{array}\right. \\
\leq & \frac{M}{360}\left\{\begin{array}{l}
\frac{1}{4}\left(15 t^{2}-15 t+3\right), \quad 0 \leq t \leq \frac{4-\sqrt{6}}{10}, \\
\frac{1}{4}\left(-5 t^{2}+t+1\right), \quad \frac{4-\sqrt{6}}{10} \leq t \leq \frac{1}{4}, \\
\frac{1}{4}\left(-13 t^{2}+11 t-1\right), \quad \frac{1}{4} \leq t \leq \frac{4+\sqrt{6}}{10} \\
\frac{1}{4}\left(7 t^{2}-5 t+1\right), \quad \frac{4+\sqrt{6}}{10} \leq t \leq 1 .
\end{array}\right.
\end{aligned}
$$

The right hand side of (36) attains its maximum at $t=0$ and $t=1$, so that

$$
\left|x^{\prime \prime}(t)\right| \leq \frac{M}{480}, \quad 0 \leq t \leq 1
$$

From (30) and (33), we have

$$
\begin{aligned}
\left|x^{\prime \prime \prime}(t)\right| \leq & \left|-18(1-t)^{2}+36 t(1-t)-6 t^{2}\right| \frac{t}{720} M+\left|\frac{-18(1-t)^{2}}{t}+18(1-t)\right| \frac{t^{2}}{720} M \\
& +\left|\frac{-9(1-t)^{2}}{t^{2}}\right| \frac{t^{3}}{360} M+\left|\frac{1}{(1-t)}\right| \frac{(1-t)^{4}}{120} M \\
= & \frac{M}{120}\left[t\left|-3+12 t-10 t^{2}\right|+t\left|-3+9 t-6 t^{2}\right|+3 t(1-t)^{2}+(1-t)^{3}\right] \\
= & \frac{M}{120} \begin{cases}\left(18 t^{3}-24 t^{2}+6 t+1\right), & 0 \leq t \leq \frac{6-\sqrt{6}}{10} \\
\left(-2 t^{3}+1\right), & \frac{6-\sqrt{6}}{10} \leq t \leq \frac{1}{2} \\
\left(-14 t^{3}+18 t^{2}-6 t+1\right), & \frac{1}{2} \leq t \leq \frac{6+\sqrt{6}}{10} \\
\left(6 t^{3}-6 t^{2}+1\right), & \frac{6+\sqrt{6} \leq t \leq 1 .}{10} \leq t \leq\end{cases}
\end{aligned}
$$

The right hand side of (37) attains its maximum at $t=\frac{4-\sqrt{7}}{9}$, so that

$$
\left|x^{\prime \prime \prime}(t)\right| \leq \frac{41+28 \sqrt{7}}{9720}, \quad 0 \leq t \leq 1 .
$$


From (31) and (33), we have

$$
\begin{aligned}
\left|x^{(4)}(t)\right| \leq & |72(1-t)-48 t| \frac{t}{720} M+\left|\frac{72(1-t)}{t}-24\right| \frac{t^{2}}{720} M+\left|\frac{36(1-t)}{t^{2}}\right| \frac{t^{3}}{360} M \\
& +\left|\frac{-8}{(1-t)^{2}}\right| \frac{(1-t)^{4}}{120} M+\left|\frac{1}{(1-t)^{3}}\right| \frac{(1-t)^{5}}{30} M \\
= & \frac{M}{30}\left[t|3-5 t|+t|3-4 t|+3 t(1-t)+3(1-t)^{2}\right] \\
= & \frac{M}{30} \begin{cases}\left(3+3 t-9 t^{2}\right), & 0 \leq t \leq \frac{3}{5}, \\
\left(3-3 t+t^{2}\right), & \frac{3}{5} \leq t \leq \frac{3}{4}, \\
\left(3-9 t+9 t^{2}\right), & \frac{3}{4} \leq t \leq 1 .\end{cases}
\end{aligned}
$$

The right hand side of (38) attains its maximum at $t=\frac{1}{6}$, so that

$$
\left|x^{(4)}(t)\right| \leq \frac{13}{120}, \quad 0 \leq t \leq 1
$$

Finally, from (32) and (33), we have

$$
\begin{aligned}
\left|x^{(5)}(t)\right| \leq & |-120| \frac{t}{720} M+\left|\frac{-120}{t}\right| \frac{t^{2}}{720} M+\left|\frac{-60}{t^{2}}\right| \frac{t^{3}}{360} M \\
& +\left|\frac{20}{(1-t)^{3}}\right| \frac{(1-t)^{4}}{120} M+\left|\frac{-5}{(1-t)^{4}}\right| \frac{(1-t)^{5}}{30} M+\left|\frac{1}{(1-t)^{5}}\right| \frac{(1-t)^{6}}{6} M \\
= & \frac{t}{2} M+\frac{1}{2}(1-t) M \\
= & \frac{1}{2} M, \quad 0 \leq t \leq 1 .
\end{aligned}
$$

This completes the proof of this Lemma.

Lemma 6. Let $x \in C^{(6)}[a, b]$, and satisfy the conditions (7). Then

$$
\begin{aligned}
|x(t)| & \leq \frac{1}{46080}(b-a)^{6} \mu, \\
\left|x^{\prime}(t)\right| & \leq \frac{159+76 \sqrt{6}}{3000000}(b-a)^{5} \mu \\
\left|x^{\prime \prime}(t)\right| & \leq \frac{1}{480}(b-a)^{4} \mu, \\
\left|x^{\prime \prime \prime}(t)\right| & \leq \frac{41+28 \sqrt{7}}{9720}(b-a)^{3} \mu, \\
\left|x^{(4)}(t)\right| & \leq \frac{13}{120}(b-a)^{2} \mu \\
\left|x^{(5)}(t)\right| & \leq \frac{1}{2}(b-a) \mu,
\end{aligned}
$$


where $\mu=\max _{0, \leq t \leq b}\left|x^{(6)}(t)\right|$. The inequalities are the best possible as the identity holds for the function $x(t)=(t-a)^{3}(b-t)^{3}$.

proof. The proof require only the transformation $\mu=a+(b-a) t, 0 \leq t \leq 1$, in Lemma 5.

\section{Proof of Theorem $\mathbb{A}$}

Suppose on the contrary that the boundary value problem (4) and (6) has a nontrivial solution $x(t)$. Then $\mu=\max _{a \leq t \leq b}\left|x^{(6)}(t)\right| \neq 0$, otherwise $x(t)$ would be a polynomial of degree $m<6$ on $[a, b]$, and $x^{(m)}(t)$ would not vanish on $[a, b]$ which cannot satisfy the boundary conditions (6).

Thus, if $\mu=\left|x^{(6)}\left(t_{1}\right)\right|$ for some $t_{1}$ in $[a, b]$, then from the differential equation (4), we have

$$
\begin{aligned}
\mu & =\left|x^{(6)}\left(t_{1}\right)\right|=\left|\sum_{i=0}^{5} p_{i}\left(t_{1}\right) x^{(i)}\left(t_{1}\right)\right| \\
& \leq \sum_{i=0}^{5}\left\|p_{i}\right\|\left|x^{(i)}\left(t_{1}\right)\right| .
\end{aligned}
$$

Now, use Lemma 3 in the above inequality, we have

$$
\begin{aligned}
\mu \leq & \frac{625}{6718464}(b-a)^{6}\left\|p_{0}\right\| \mu+\frac{1}{720}(b-a)^{5}\left\|p_{1}\right\| \mu \\
& +\frac{1}{72}(b-a)^{4}\left\|p_{2}\right\| \mu+\frac{1}{12}(b-a)^{3}\left\|p_{3}\right\| \mu \\
& +\frac{1}{3}(b-a)^{2}\left\|p_{4}\right\| \mu+\frac{5}{6}(b-a)\left\|p_{5}\right\| \mu \\
= & \theta \mu .
\end{aligned}
$$

We note that at least one of the numbers $\left\|p_{i}\right\|, 0 \leq i \leq 5$, is different from zero, otherwise, again $x(t)$ would be a polynomial of degree less than 6 , and cannot satisfy the boundary conditions (6). Hence, it is necessary that $\theta \geq 1$.

This completes the proof of theorem A.

The proof of theorem $B$ is similar to that of Theorem $A$. We omit the detail.

\section{References}

1. G.A. Bogar and G.B. Gustafson, "Effective estimates of invertibility intervals for linear multipoint boundary value problems," J. Differential Equations 29(1978), 180-204.

2. R.P. Agarwal, G.V. Milovanovic, "On an inequality of Bogar and Gustafson," J. math. anal. appl. $146,207-216(1990)$. 